\title{
BMJ Open Risk factors for recurrent falls in older adults: a study protocol for a systematic review with meta-analysis
}

\author{
Deborah A Jehu (D , 1,2,3 Jennifer C Davis, ${ }^{2,4}$ Teresa Liu-Ambrose ${ }^{1,2,3}$
}

To cite: Jehu DA, Davis JC, Liu-Ambrose T. Risk factors for recurrent falls in older adults: a study protocol for a systematic review with meta-analysis. BMJ Open 2020;10:e033602. doi:10.1136/ bmjopen-2019-033602

- Prepublication history and additional material for this paper are available online. To view these files, please visit the journal online (http://dx.doi. org/10.1136/bmjopen-2019033602).

Received 12 August 2019 Revised 17 December 2019 Accepted 28 January 2020

Check for updates

(C) Author(s) (or their employer(s)) 2020. Re-use permitted under CC BY-NC. No commercial re-use. See rights and permissions. Published by BMJ.

For numbered affiliations see end of article.

Correspondence to Dr Teresa Liu-Ambrose; teresa.ambrose@ubc.ca

\begin{abstract}
Introduction Older adults who fall recurrently (i.e., >1 fall/ year) are at risk for functional decline and mortality. Key risk factors for recurrent falls in community-dwelling older adults are not well established due to methodological limitations, such as recall bias. A better understanding of the risk factors for recurrent falls will aid in refining clinical practice guidelines for secondary fall prevention strategies. The primary objective of this systematic review with metaanalysis is to examine the risk factors for recurrent falls in prospective studies among community-dwelling older adults.
\end{abstract}

Methods and analysis A comprehensive search for articles indexed in MEDLINE, EMBASE, PsycINF0 and CINAHL databases as well as grey literature was conducted on April 25, 2019. We will use MeSH and keyword search terms around the following topics: falls, recurrence, fall-risk, ageing and prospective studies. Prospective studies with monthly falls monitoring for 12 months, investigating risk factors for recurrent falls in older adults will be included. One author will complete the search. Two authors will remove duplicates and screen the titles and abstracts for their potential inclusion against the eligibility criteria. Two authors will screen the full texts and extract the data using a piloted extraction sheet. Included studies will be evaluated for the risk of bias with the Joanna Briggs Institute Prevalence Critical Appraisal tools. The quality of reporting will be determined with the Strengthening the Reporting of OBservational studies in Epidemiology. The data extraction will include study characteristics as well as sociodemographic, balance and mobility, sensory and neuromuscular, psychological, medical, medication and environmental factors. The results will be presented via figures, summary tables, metaanalysis (when possible) and narrative summaries.

Ethics and dissemination No ethics approval will be required. Findings will be disseminated through publication and media.

PROSPERO registration number CRD42019118888;

Pre-results.

\section{INTRODUCTION}

Falls are a major health concern and the second leading cause of unintentional injury deaths. ${ }^{1}$ Older adults who fall more than once per year are defined as recurrent fallers. ${ }^{2-7}$ Recurrent fallers are at higher risk for morbidity and mortality than non-fallers and

\section{Strengths and limitations of this study}

This systematic review with meta-analysis will identify risk factors for recurrent falls.

- The heterogeneity of fall-risk factors as well as the possible poor quality of included reports, which may limit the ability to generate conclusions based on high confidence.

- This study is limited by imprecision in the pooled effect estimate due to possible small sample sizes in some studies.

- This study may represent a healthier sample of recurrent fallers as a result of studies with significant loss to follow-up.

single fallers. ${ }^{7-17}$ Thus, secondary prevention is critical for minimising functional decline and maintaining quality of life. ${ }^{14} \mathrm{~A}$ better understanding of risk factors for recurrent falls is needed to develop effective secondary fall prevention strategies.

Three systematic reviews have examined risk factors for recurrent falls among community-dwelling older adults but they included retrospective fall data. ${ }^{18-20}$ Retrospective studies are prone to more recall bias compared with prospective studies, with $5.8 \%$ of non-fallers, $32.8 \%$ of single fallers and up to $50 \%$ of recurrent fallers deny falling in the previous year when comparing retrospective to prospective falls monitoring. ${ }^{21}$ One of these reviews only searched the literature between 2010 and $2014,{ }^{20}$ greatly limiting the scope of published evidence. The strength of the evidence in two of the three reviews is difficult to determine because assessment of study quality and risk of bias were not performed. ${ }^{19} 20$ Thus, there is a need for a systematic review of data solely from prospective studies (of falls) with an evaluation of study quality and risk of bias. Moreover, female $\operatorname{sex}^{22}$ and greater dependence ${ }^{23}$ have increased fall-risk factors and falls, thus they should be considered in analyses. 
The risk factors unique to recurrent falls remain unclear. Therefore, the purpose of this study is to conduct a systematic review with meta-analysis of the risk factors for recurrent falls solely in prospective studies. Secondary research questions include (1) do these risk factors vary as a function of biological sex? and 2) do these risk factors vary as a function of living situation? Another secondary aim is to conduct sensitivity analyses to compare studies with low versus high risk of bias to draw accurate conclusions on risk factors for recurrent falls.

\section{METHODS \\ Design}

The current review methods followed the Preferred Reporting Items for Systematic Reviews and Meta-Analysis Protocols (PRISMA-P; online supplementary file 1$)^{24}$ and Meta-Analysis of Observational Studies in Epidemiology guidelines. $^{25}$

\section{Inclusion criteria}

Types of studies

All prospective quantitative studies examining risk factors contributing to recurrent falls in older adults published in English or French will be included. Prospective falls data must be collected at least monthly (eg, phone calls, fall calendars) for 12 months to be included in this study. In case of multiple publications for one study, all articles will be used to obtain maximum information.

\section{Types of participants}

Studies will be included if participants: (1) are 60 years of age and older or are a mean age of 65 years and older; (2) are residing in the community, residential care or clinical environments; and (3a) are recurrent fallers having sustained two or more falls in the first prospective year following the initial assessment; or (3b) have had a documented fall(s) in the previous 6 months and are being followed prospectively for 6 months for subsequent fall(s). Note that a fall will be defined as 'an unexpected event in which the participant comes to rest on the ground, floor or lower level'. ${ }^{26}$ A recurrent faller will be defined as a person falling more than once in a 12-month prospective period. ${ }^{2-7}$ Risk will be defined as 'the probability that an unwanted health event (eg, a future fall) will occur'. ${ }^{27}$

\section{Exclusion criteria}

Studies will be excluded if they: (1) focus on patient groups (eg, neurodegenerative diseases) to promote a more homogeneous sample; (2) only include retrospective falls data; (3) do not separate single fallers from recurrent fallers; and 4) are not prospective (eg, crosssectional studies, reviews and meta-analyses). Finally, we will contact authors to inquire about fall outcome data that are not reported and give authors 4 weeks to respond.

\section{Search strategy}

The following electronic databases will be searched: MEDLINE (Ovid interface; 1946-2019), EMBASE (Ovid interface: 1974-2019), PsycINFO (Ebsco interface: 15972019) and CINAHL (Ebsco interface: 1982-2019) on 25 April 2019. The search strategy for each database is provided in online supplementary file 1 . We will manually search bibliographies of articles included in the review, the references of other study reviews that match our inclusion criteria, trial registries and articles citing our eligible articles in Google Scholar and Web of Science. Conference proceedings will also be searched from the International Society for Posture and Gait Research between 1971 and 2019. We will also consult with content experts in the field to determine whether we have included all relevant articles (figure 1). ${ }^{24}$

\section{Study selection}

One author will complete the search and remove the duplicates. Two authors will screen the titles and abstracts for their potential inclusion against the eligibility criteria, screen the full texts and extract the data using a pretested extraction sheet. After screening and data extraction is complete, the reviewers will compare their extracted data. In case of discrepancies, discussion will be held until consensus is reached. A third review team member will be consulted if agreement cannot be reached. The PRISMA flow diagram will be constructed after the screening process. Excluded articles will be listed in online supplementary file 1.

\section{Outcome measures and data extraction}

The primary outcome measure will be the rate of prospective falls because frequent fallers generally exhibit more fall-risk factors and prospective falls study timelines are heterogeneous. ${ }^{18}$ The fall rate per year will be calculated for each study using the following formula:

$$
\text { Fall rate }=\frac{\text { average number of falls per participant }}{\text { days in the follow }- \text { up time period }} \times 365 \text { days }
$$

Based on a fall-risk classification system, ${ }^{28}$ the data extraction sheet will be comprised of the following secondary outcomes.

- Study characteristics: that is, article citation, country where the study was conducted, setting (community, hospital and long-term care), source of funding and declarations of interest of the primary researchers.

- Sociodemographical factors: for example, age, sex, fall characteristics (eg, number of recurrent fallers, frequency retrospective and prospective falls, timeline of retrospective and prospective falls, method of collecting fall data, rate of falls, severity of injury(ies), time to first injury and fall-risk).

- Balance and mobility factors: for example, Tinetti Balance \& Gait Assessment, ${ }^{29}$ Timed Up and Go Test $(\mathrm{s}),{ }^{30}$ gait speed $(\mathrm{m} / \mathrm{s})$, sit-to-stand (s) and sway area $\left(\mathrm{mm}^{2}\right)$.

- Sensory and neuromuscular factors: for example, visual impairment (eg, cataracts, glaucoma; \%), contrast sensitivity $(\mathrm{dB})$, vision score, hearing impairment $(\%)$, pain $(\%)$, proprioceptive function $\left({ }^{\circ}\right.$ 


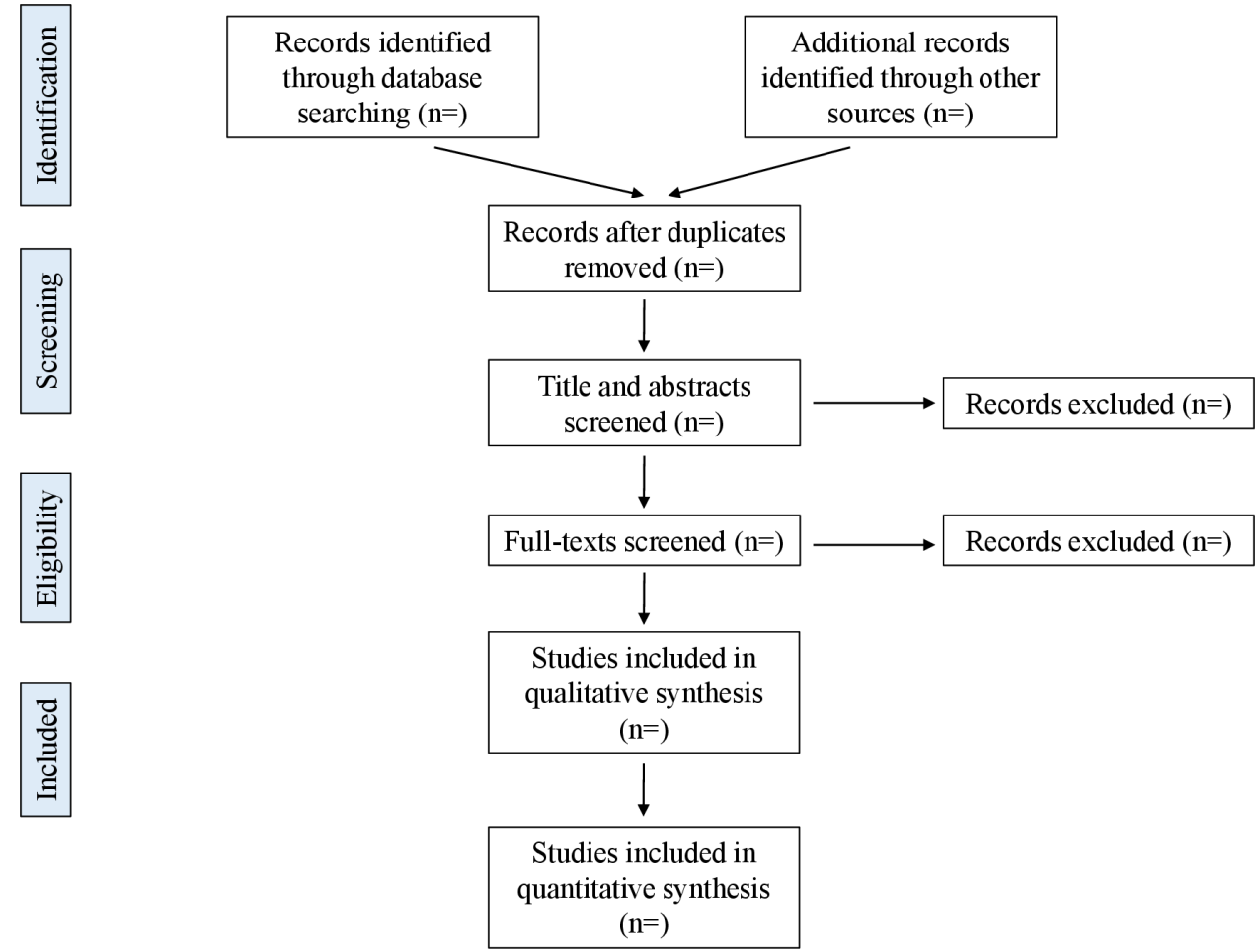

Figure 1 Preferred Reporting Items for Systematic Reviews and Meta-Analysis flow diagram of the identification, screening and eligibility of included articles.

error), dizziness $(\%)$, reaction time (ms) and muscle strength $\left(\mathrm{N} / \mathrm{m}^{2}\right)$.

- Psychological factors: for example, Mini-Mental State Examination, ${ }^{31}$ Trail Making test, ${ }^{32}$ fear of falling, Activities-specific Balance Confidence scale, ${ }^{33}$ Geriatric Depression Scale ${ }^{34}$ dual-tasking and Central Nervous System measures (eg, MRI).

- Medical factors: comorbidities (\% of those with none, $1,2$ or $>2)$.

- Medication use: Medications known to influence falls or fall-risk factors (eg, antidepressants, sedatives, diuretics and vitamin $\mathrm{D}$; \% of sample).

- Environmental factors: for example, footwear (\% of those with appropriate vs inappropriate shoes), weather conditions ( $\%$ of falls in slippery, wet, loose gravel and dry conditions), time of day ( $\%$ of falls in the morning vs evening) and location (\% of falls indoors vs outdoors).

When possible, the means of these outcomes will be collected at baseline before prospective falls. Regression coefficients, standard errors, ORs and relative risk will also be extracted. We will examine any retraction statements and errata, which are associated with included studies and, where applicable, update the recorded data accordingly.

\section{Missing data management}

Missing fall-risk classification outcome data will be requested from authors. If missing data still exists, the analysis will be conducted on the final available data. The potential impact of missing data on the review findings will be addressed in the final report.

\section{Quality of reporting}

Researchers and clinicians are often constrained with reports of studies including inadequate detail of information for data synthesis and implementation of findings. ${ }^{35}$ Given the human and financial resources required to conduct clinical studies, inadequate reporting has ethical and moral implications. ${ }^{36}$ The Strengthening the Reporting of OBservational studies in Epidemiology (STROBE) checklist was designed to guide researchers in the quality of reporting ${ }^{37}$ and has shown good reliability, ${ }^{38}$ however it was not designed to evaluate the quality of reporting. ${ }^{39}$ To our knowledge, there are no dedicated or validated tools to assess the quality of reporting. Therefore, two authors will determine the reporting quality of the included articles using the STROBE to guide the interpretation of our findings. A study will be deemed as high quality if most criteria are met, while low quality will be assigned if few criteria are met. Reporting quality data from each study will be presented in tables and/or figures by STROBE tool items.

\section{Risk of bias assessment}

Two independent reviewers will assess the risk of bias of each study using the Joanna Briggs Institute Prevalence Critical Appraisal tool and compare their results for any discrepancies. This tool was developed to assess the methodological quality of studies reporting prevalence data to be included in systematic reviews. ${ }^{40}$ Using the Joanna Briggs Institute Prevalence Critical Appraisal Tool, a low risk of bias will be assigned to each eligible study if most methodological criteria are met, while a high risk of bias will be assigned for studies with few criteria are met. Disagreements between reviewers will be resolved through discussion with a third 
neutral reviewer on the team. In the discussion, a greater emphasis will be placed on studies with a low risk of bias. The risk of bias data from each study will be presented in tables and/or figures by Joanna Briggs Institute Prevalence Critical Appraisal tool items.

\section{Data synthesis and summary of results}

The findings from the study will be presented in a narrative synthesis, structured around the fall-risk outcome measures and presented in tables and figures. A table of study characteristics will be displayed including the following variables: citation, sample size, $\%$ female, mean age and duration of prospective falls follow-up.

Where population and outcome of the studies are clinically, methodologically and statistically similar, a metaanalysis will be done by pooling appropriate data and using RefWorks V.2.0. If enough data support a quantitative synthesis of fall-risk outcomes, a random-effects metaanalysis will be performed on the pooled data given the potential heterogeneity in participant characteristics.

The $\mathrm{I}^{2}$ test will be used to test the statistical heterogeneity among all studies as well as visual inspections of forest plots. We will consider a significance level less than 0.10 as evidence of heterogeneity. Substantial statistical (ie, $\mathrm{I}^{2} \geq 50 \%$ ) or methodological heterogeneity (eg, differences in fall-risk factors explored and variation in the tools used to measure fall-risk factors) may impact fall-risk factors. ${ }^{41}$ A priori subgroup analyses will be explored by comparing important participant factors between studies (eg, age, sex, clinical condition, place of residence and number of recurrent falls) and study risk of bias factors (eg, adequate adjustment of confounding variables and losses of prospective falls calendars) to reveal potential sources of heterogeneity. Sensitivity analyses will be conducted to compare results with low versus high risk of bias. Studies will not be included in the meta-analysis if insufficient data are provided or if they have a high risk of bias. Funnel plot asymmetry will be conducted using a rank correlation between fall-risk factors effects and their SE. ${ }^{42}$ A minimum of 10 studies will be included in funnel plot analyses to ensure that the test power is high enough to distinguish chance from real asymmetry. ${ }^{41}$ If asymmetry occurs, we will consider reporting bias, poor methodological quality, true heterogeneity, artefactual and chance factors during interpretation. ${ }^{41}$

\section{Data statement}

The dataset generated from this study will be available in the published article.

\section{Patient and public involvement}

Patients will not be involved in the design of this study protocol, as no participant recruitment will be necessary.

\section{Amendments}

Should amendments to this protocol be necessary, they will be documented on PROSPERO.

\section{DISCUSSION, ETHICS AND DISSEMINATION}

Many falls are preventable; ${ }^{43}$ however, less is known about how to prevent falls among those at a high fall-risk. Thus, a better identification of risk factors for recurrent falls would inform best practices for fall-risk assessment and secondary fall prevention interventions. These results may benefit clinicians in better assessing and treating their clients, older adults and their caregivers by receiving best practice care, and policy makers with promoting and implementing best practice care. Should the eligible articles present with substantial heterogeneity in risk factors for recurrent falling, we will report the sources of heterogeneity in the results and discuss their impact on the review.

No ethics approval will be required. The results of this systematic review and meta-analysis will be disseminated via peer-reviewed publication and conference presentation.

\section{Author affiliations}

${ }^{1}$ Djavad Mowafaghian Centre for Brain Health, Vancouver Coastal Health Research Institute, Vancouver, British Columbia, Canada

${ }^{2}$ Centre for Hip Health and Mobility, Vancouver Coastal Health Research Institute, Vancouver, British Columbia, Canada

${ }^{3}$ Aging, Mobility and Cognitive Neuroscience Laboratory, Department of Physical

Therapy, University of British Columbia, Vancouver, British Columbia, Canada

${ }^{4}$ Social \& Economic Change Laboratory, Faculty of Management, University of

British Columbia - Okanagan Campus, Kelowna, British Columbia, Canada

Acknowledgements A special thank you to Charlotte Beck, who is a librarian and was instrumental in providing advice on structuring the keyword search, as well as to Dr Carole Lunny, who provided important methodological considerations when conducting systematic reviews with meta-analyses.

Contributors TLA, JCD and DAJ developed the research question. DAJ registered the protocol and wrote the first draft of the manuscript. DAJ and JCD developed the search strategy and created the data extraction form. JCD and TLA provided critical review and feedback at each stage of the process. All authors critically reviewed and edited the final manuscript.

Funding Dr Liu-Ambrose is a Canada Research Chair (Tier 2) in Physical Activity, Mobility, and Cognitive Neuroscience, Department of Physical Therapy, University of British Columbia. Dr. Jehu is a funded postdoctoral fellow through the Michael Smith Foundation for Health Research. The funders played no role in the design of this study.

Competing interests None declared.

Patient consent for publication Not required.

Provenance and peer review Not commissioned; externally peer reviewed.

Open access This is an open access article distributed in accordance with the Creative Commons Attribution Non Commercial (CC BY-NC 4.0) license, which permits others to distribute, remix, adapt, build upon this work non-commercially, and license their derivative works on different terms, provided the original work is properly cited, appropriate credit is given, any changes made indicated, and the use is non-commercial. See: http://creativecommons.org/licenses/by-nc/4.0/.

ORCID iD

Deborah A Jehu http://orcid.org/0000-0002-9084-7445

\section{REFERENCES}

1 WHO. Falls fact sheet. Available: https://www.who.int/news-room/ fact-sheets/detail/falls [Accessed 16 Jan 2018].

2 Coleman AL, Cummings SR, Yu F, et al. Binocular visual-field loss increases the risk of future falls in older white women. J Am Geriatr Soc 2007;55:357-64.

3 Ensrud KE, Ewing SK, Taylor BC, et al. Frailty and risk of falls, fracture, and mortality in older women: the study of osteoporotic fractures. J Gerontol A Biol Sci Med Sci 2007;62:744-51. 
4 Knudtson MD, Klein BEK, Klein R. Biomarkers of aging and falling: the Beaver dam eye study. Arch Gerontol Geriatr 2009;49:22-6.

5 Panzer VP, Wakefield DB, Hall CB, et al. Mobility assessment: sensitivity and specificity of measurement sets in older adults. Arch Phys Med Rehabil 2011;92:905-12.

6 Stone KL, Ancoli-Israel S, Blackwell T, et al. Actigraphy-measured sleep characteristics and risk of falls in older women. Arch Intern Med 2008;168:1768-75.

7 Kelsey JL, Procter-Gray E, Berry SD, et al. Reevaluating the implications of recurrent falls in older adults: location changes the inference. J Am Geriatr Soc 2012;60:517-24.

8 Muir SW, Berg K, Chesworth B, et al. Quantifying the magnitude of risk for balance impairment on falls in community-dwelling older adults: a systematic review and meta-analysis. J Clin Epidemiol 2010;63:389-406

9 Nevitt MC, Cummings SR, Kidd S, et al. Risk factors for recurrent nonsyncopal falls. A prospective study. JAMA 1989;261:2663-8.

10 Muir SW, Gopaul K, Montero Odasso MM. The role of cognitive impairment in fall risk among older adults: a systematic review and meta-analysis. Age Ageing 2012;41:299-308.

11 Stubbs B, Schofield P, Binnekade T, et al. Pain is associated with recurrent falls in community-dwelling older adults: evidence from a systematic review and meta-analysis. Pain Med 2014;15:1115-28.

12 de Vries M, Seppala LJ, Daams JG, et al. Fall-risk-increasing drugs: a systematic review and meta-analysis: I. cardiovascular drugs. J Am Med Dir Assoc 2018;19:371.e1-371.e9.

13 Seppala LJ, Wermelink AMAT, de Vries M, et al. Fall-risk-increasing drugs: a systematic review and meta-analysis: II. psychotropics. $J$ Am Med Dir Assoc 2018;19:371.e11-371.e17.

14 Tinetti ME, Williams CS. The effect of falls and fall injuries on functioning in community-dwelling older persons. J Gerontol A Biol Sci Med Sci 1998;53:M112-9.

15 Soares WJS, Lopes AD, Nogueira E, et al. Physical activity level and risk of falling in community-dwelling older adults: systematic review and meta-analysis. J Aging Phys Act 2018:1-10.

16 Kiel DP, O'Sullivan P, Teno JM, et al. Health care utilization and functional status in the aged following a fall. Med Care 1991;29:221-8

17 Wolinsky FD, Johnson RJ, Fitzgerald JF. Falling, health status, and the use of health services by older adults. A prospective study. Med Care 1992;30:587-97.

18 Lusardi MM, Fritz S, Middleton A, et al. Determining risk of falls in community dwelling older adults: a systematic review and meta-analysis using posttest probability. J Geriatr Phys Ther 2017;40:1-36.

19 Deandrea S, Lucenteforte E, Bravi F, et al. Risk factors for falls in community-dwelling older people: a systematic review and metaanalysis. Epidemiology 2010;21:658-68.

20 Sousa LMM, Marques-Vieira CMA, Caldevilla MNGNde, et al. Risk for falls among community-dwelling older people: systematic literature review. Rev Gaucha Enferm 2017;37:e55030.

21 Garcia PA, Dias JMD, Silva SLA, et al. Prospective monitoring and self-report of previous falls among older women at high risk of falls and fractures: a study of comparison and agreement. Braz J Phys Ther 2015;19:218-26.

22 Gale CR, Cooper C, Aihie Sayer A. Prevalence and risk factors for falls in older men and women: the English longitudinal study of ageing. Age Ageing 2016;45:789-94.
23 Gill TM, Murphy TE, Gahbauer EA, et al. Association of injurious falls with disability outcomes and nursing home admissions in community-living older persons. Am J Epidemiol 2013;178:418-25.

24 Shamseer L, Moher D, Clarke M, et al. Preferred reporting items for systematic review and meta-analysis protocols (PRISMA-P) 2015 elaboration and explanation. BMJ 2015;349:97647.

25 Stroup DF, Berlin JA, Morton SC, et al. Meta-Analysis of observational studies in epidemiology: a proposal for reporting. meta-analysis of observational studies in epidemiology (moose) group. JAMA 2000;283:2008-12.

26 Lamb SE, Jørstad-Stein EC, Hauer K, et al. Development of a common outcome data set for fall injury prevention trials: the prevention of falls network Europe consensus. J Am Geriatr Soc 2005;53:1618-22.

27 Murray C, Lopez A. World Health Report 2002: Reducing risks and promoting health lifestyle. Geneva, Switzerland: World Health Organization, 2002.

28 Lord SR, Sherrington C, Menz HB, et alPress CU, ed. Falls in older people: risk factors and strategies for prevention. 2nd, 2007: 1-160.

29 Tinetti ME. Performance-oriented assessment of mobility problems in elderly patients. J Am Geriatr Soc 1986;34:119-26.

30 Browne W, Nair BKR. The timed up and go test. Med J Aust 2019;210:13.

31 Folstein MF, Folstein SE, McHugh PR. "Mini-mental state". J Psychiatr Res 1975;12:189-98.

32 Spreen O, Strauss E. A compendium of neurological tests. 2edn. New York: Oxford University Press, Inc, 1998.

33 Myers AM, Fletcher PC, Myers AH, et al. Discriminative and evaluative properties of the activities-specific balance confidence (ABC) scale. J Gerontol A Biol Sci Med Sci 1998;53:M287-94.

34 Yesavage JA, Brink TL, Rose TL, et al. Development and validation of a geriatric depression screening scale: a preliminary report. $J$ Psychiatr Res 1982;17:37-49.

35 Glasziou P, Meats E, Heneghan C, et al. What is missing from descriptions of treatment in trials and reviews? BMJ 2008;336:1472-4.

36 Moher D. Reporting research results: a moral obligation for all researchers. Can J Anaesth 2007;54:331-5.

37 von Elm E, Altman DG, Egger M, et al. Strengthening the reporting of observational studies in epidemiology (STROBE) statement: guidelines for reporting observational studies. BMJ 2007;335:806-8.

38 Brosseau L, Guitard P, Laroche C, et al. La version francocanadienne du « STrengthening the Reporting of OBservational studies in Epidemiology » (STROBE) Statement : L'outil STROBE. Physiotherapy Canada 2019;71:1-10.

39 da Costa BR, Cevallos M, Altman DG, et al. Uses and misuses of the STROBE statement: bibliographic study. BMJ Open 2011;1:e000048.

40 Munn Z, Moola S, Riitano D, et al. The development of a critical appraisal tool for use in systematic reviews addressing questions of prevalence. Int J Health Policy Manag 2014;3:123-8.

41 Sterne JAC, Sutton AJ, loannidis JPA, et al. Recommendations for examining and interpreting funnel plot asymmetry in meta-analyses of randomised controlled trials. BMJ 2011;343:d4002.

42 Begg CB, Mazumdar M. Operating characteristics of a RANK correlation test for publication bias. Biometrics 1994:50:1088-101.

43 Liu-Ambrose T, Donaldson MG, Ahamed Y, et al. Otago home-based strength and balance retraining improves executive functioning in older fallers: a randomized controlled trial. J Am Geriatr Soc 2008;56:1821-30. 